\title{
Pancreatitis aguda y necrosis pancreática: conceptos actuales y tratamiento
}

\author{
Acute pancreatitis and pancreatic necrosis: current concepts and treatment \\ Rodrigo Velázquez de la Vega, ${ }^{*}$ Luis Eduardo Cárdenas Lailson**
}

\begin{abstract}
Palabras clave: Pancreatitis aguda, necrosis pancreática, complicaciones, tratamiento, cirugía de invasión mínima.

Key words: Acute pancreatitis, pancreatic necrosis, complications, treatment, minimally invasive surgery.
\end{abstract}

\section{RESUMEN}

La pancreatitis aguda es un proceso inflamatorio del páncreas con afección local y sistémica, con alta prevalencia a nivel mundial. La causa más frecuente es biliar. Su diagnóstico se establece con dos de los tres criterios. En su abordaje debe identificarse la severidad para optimizar el tratamiento, el cual inicialmente es médico, pero puede requerirse tratamiento invasivo, dependiendo de la evolución que presente y es necesario estandarizarlo para lograr una adecuada evolución y disminuir la morbimortalidad. El objetivo de este artículo es dar a conocer los conceptos actuales en relación con la pancreatitis aguda, así como los puntos más importantes del tratamiento de esta patología que permiten establecer criterios y de esta manera ofrecer a los pacientes un tratamiento protocolizado.

\section{ABSTRACT}

Acute pancreatitis is an inflammatory process of the pancreas with local and systemic affection, with a high prevalence worldwide. The most common cause is biliary. It is diagnosed using two of the three criteria. Within its approach, severity must be identified in order to optimize treatment. Treatment is initially medical, but may require invasive treatment depending on its course. Its treatment requires standardization in order to have an adequate evolution and decrease morbidity and mortality. The objective of this article is to present the current concepts related to acute pancreatitis, as well as the most important points of the treatment of this pathology, allowing establishing criteria, and thus offering a protocolized treatment to patients.

\section{ANTECEDENTES HISTÓRICOS}

$\mathrm{H}^{2}$ ace más de 120 años Reginald H. Fitz describió por primera vez la pancreatitis. Tanto en Estados Unidos como en Europa el tratamiento que se utilizaba para la necrosis pancreática era la pancreatectomía. En 1929 se inició la medición de amilasa, en la que se detectó la existencia de pancreatitis sin necrosis, la cual resolvía sin ninguna intervención quirúrgica, por lo que el tratamiento de la pancreatitis se modificó, siendo el tratamiento médico el tratamiento de elección. Sin embargo, se observó que los casos más graves continuaban muriendo a pesar del manejo médico. En 1959 Pallock realizaba resecciones pancreáticas en pacientes muy graves, posteriormente otros cirujanos como Rives, Devic y Hollender siguieron la misma conducta justificando dicho procedimiento con el que se reducía la mortalidad de 100 a 60\%. En 1985
Beger determinó con el uso de la tomografía que la resección debía limitarse únicamente al tejido necrótico, preservando el tejido sano. Desde entonces se han desarrollado diversas técnicas con distintos resultados para los casos de necrosis pancreática. ${ }^{1}$

\section{EPIDEMIOLOGÍA}

La pancreatitis aguda es una patología abdominal muy frecuente. Se estima que tiene una incidencia de 4.9-73.4:100,000 habitantes a nivel mundial. ${ }^{2}$ La causa más frecuente en el mundo es la pancreatitis biliar (32-49\%) seguida de la alcohólica en segundo lugar (20-31.8\%). ${ }^{3}$ En México, según un estudio publicado en 2004, se identificó la pancreatitis biliar como la principal causa en 51\% seguida de la pancreatitis alcohólica en 39\% y otras causas en $10 \% .{ }^{4}$ A nivel mundial se ha observado que la etiología no se identifica hasta en $23.2 \% .^{5}$ 


\section{FISIOPATOLOGÍA}

Se han identificado dos fases. La primera inicia con la activación de los gránulos zimógenos dentro de los acinos pancreáticos. Éstos se unen a lisozimas intracelulares como catepsina B, convirtiendo el tripsinógeno en tripsinas y de esta manera se liberan enzimas pancreáticas hacia los acinos y entre las células de los acinos. Una vez activadas las enzimas pancreáticas se induce respuesta inflamatoria local por medio de neutrófilos, macrófagos y factores proinflamatorios como FNT alfa, IL-6 e IL-8. Posteriormente la respuesta inflamatoria se generaliza, produciendo una respuesta inflamatoria sistémica que puede ocasionar falla orgánica. ${ }^{6}$ Es posible que esta fase dure hasta siete días.

En la segunda fase hay manifestaciones locales a consecuencia de la respuesta inflamatoria local. Las manifestaciones locales pueden ser colecciones pancreáticas o peripancreáticas y la necrosis, pancreática o peripancreática. ${ }^{2}$

\section{CLASIFICACIÓN}

En el año 2012 se hizo una revisión de la clasificación de la pancreatitis, en la cual se dividió la pancreatitis aguda en edematosa intersticial y necrosante. ${ }^{7}$ En la pancreatitis edematosa intersticial pueden surgir dos complicaciones locales: las colecciones agudas (menos de cuatro semanas) y el pseudoquiste (más de cuatro semanas). A su vez, las complicaciones que se presentan en la pancreatitis necrosante son colección necrótica aguda (menos de cuatro semanas) y necrosis sin pared "WON" (más de cuatro semanas). A partir de esta clasificación se han eliminado términos que se usaban previamente y que no eran claros. ${ }^{8}$

\section{DIAGNÓSTICO}

El diagnóstico de pancreatitis aguda se establece con base en al menos dos de los tres criterios: dolor abdominal sugestivo de pancreatitis aguda, elevación de la amilasa y lipasa tres veces el límite superior del rango normal mediante estudios de imagen (TAC o RM). El uso de estos estudios se limita únicamente cuando hay sospecha diagnóstica o el paciente no presenta mejoría después de 48-72 horas de tratamiento para descartar complicaciones locales. ${ }^{2}$

En el protocolo de estudio de los pacientes con pancreatitis es necesario establecer la etiología, por lo que a todo paciente con pancreatitis aguda se le debe hacer un interrogatorio completo para conocer antecedentes de colelitiasis, consumo de alcohol, dislipidemia, antecedente familiar de pancreatitis, uso de medicamentos, infecciones virales y en casos muy raros mordedura o picadura de insecto.

Debido a que la etiología más frecuente es la biliar, debe realizarse ultrasonido de hígado y vías biliares a todos los pacientes, en caso de ser negativo para colelitiasis y se descarte etiología etílica, es necesario buscar otras causas como hipertrigliceridemia, hipercalcemia viral, alteraciones anatómicas autoinmunes, entre otras. Según los indicadores internacionales, no debe haber más de $20 \%$ de casos de pancreatitis cuya etiología no se identifique. ${ }^{5}$

\section{SEVERIDAD}

Actualmente la pancreatitis aguda se clasifica con base en su severidad en tres grados de acuerdo con lo establecido en la revisión de los criterios de Atlanta en 2012; se divide en pancreatitis aguda leve, pancreatitis aguda moderadamente severa y pancreatitis aguda severa.

La pancreatitis leve es aquélla en la que el paciente no evidencia fallas ni complicaciones locales. La pancreatitis moderada presenta fallas (respiratoria, renal o cardiovascular con base en los criterios de Marshall modificados) que revierten en menos de 48 horas con o sin complicaciones locales y la pancreatitis severa evidencia fallas que no revierten en menos de 48 horas. ${ }^{6}$

Otras escalas utilizadas previamente para determinar la severidad de la pancreatitis son APACHE II, los criterios de Ranson, SOFA, BISAP y BISAP-O, entre otras; sin embargo, algunas de estas escalas presentan mayor complejidad para obtener la puntuación.

\section{TRATAMIENTO}

El tratamiento inicial en pancreatitis aguda tiene como objetivo garantizar una adecuada perfusión de los tejidos, controlar el dolor y evitar complicaciones. Debe realizarse un abordaje 
protocolizado y ordenado. Se sugiere utilizar la nemotecnia PANCREAS ${ }^{9}$ ( $\mathrm{P}$ perfusión, A analgesia, $\mathrm{N}$ nutrición, $\mathrm{C}$ clínica, $\mathrm{R}$ radiología, E CPRE, A antibiótico, $\mathrm{S}$ cirugía).

Para mantener una adecuada perfusión se requiere aportar soluciones por vía intravenosa, debe medirse la diuresis de $0.5 \mathrm{ml} / \mathrm{kg} / \mathrm{h}$. Se recomienda el uso de solución Hartmann, ya que contiene menor cantidad de cloro, se requieren volúmenes altos y se disminuye el riesgo de acidosis metabólica hiperclorémica. Aportar oxígeno suplementario para mantener una saturación mayor de $95 \%$ es otra de las medidas para una adecuada perfusión. ${ }^{10}$

El control del dolor es fundamental en el manejo de la pancreatitis aguda. La severidad de la pancreatitis se relaciona con la intensidad y duración del dolor, mientras que en una pancreatitis edematosa intersticial el dolor puede llegar a ser controlado con analgésico, en una necrosis pancreática el dolor puede llegar a ser tan intenso que requiere el uso de opiáceos potentes. Se sugiere utilizar la escala de dolor propuesta por la OMS para el control del dolor en la pancreatitis. Estudios revelan que la buprenorfina tiene alta eficacia y el uso de opioides no está contraindicado. ${ }^{11} \mathrm{Se}$ ha propuesto el uso de bloqueo torácico epidural como analgesia y se ha constatado que además de aliviar el dolor, mejora la perfusión, disminuye el daño por isquemia y la respuesta inflamatoria es mediada por neuronas. ${ }^{12}$

El ayuno está indicado en todos los pacientes, debe llevarse el menor tiempo posible (iniciar dieta en las primeras 24 horas de hospitalización) para evitar la desnutrición y la translocación bacteriana. ${ }^{13}$ Se recomienda iniciar con dieta blanda baja en lípidos, ya que se ha demostrado que de esta manera se acorta el tiempo de estancia hospitalaria y el aporte nutricio es mayor comparado con la dieta líquida y no aumenta la necesidad de suspender la dieta por dolor. ${ }^{14}$ Lo anterior se ha detectado en la pancreatitis leve.

En pacientes con pancreatitis severa se ha comprobado que no hay diferencia en la evolución en pacientes que requieren apoyo enteral, ya sea con sonda nasogástrica o sonda nasoyeyunal. Debido a que la colocación de la sonda nasogástrica no requiere guía endoscópica o fluoroscópica, la dieta enteral con sonda nasogástrica puede iniciarse antes. La nutrición parenteral debe reservarse para pacientes en quienes no es posible iniciar vía enteral, ya sea por vía oral o por sonda en un periodo de cinco a siete días, pues se ha observado en múltiples estudios que iniciar con dieta enteral acorta el tiempo de estancia hospitalaria, disminuye el riesgo de complicaciones infecciosas así como la mortalidad en pancreatitis severa. ${ }^{13}$ La nutrición parenteral temprana se reserva para pacientes que sufren de desnutrición desde su ingreso y no es posible iniciar vía enteral. ${ }^{15}$

En pacientes que de forma concomitante tienen signos de colangitis, se requiere colangiopancreatografía retrógrada endoscópica (CPRE) en las primeras 24 horas a partir de su ingreso. En pacientes con riesgo intermedio de coledocolitiasis se recomienda ultrasonido endoscópico o colangiorresonancia. ${ }^{16}$ En nuestra institución se realiza CPRE a los pacientes con pancreatitis y con alto riesgo de coledocolitiasis. Si el paciente presenta riesgo intermedio de coledocolitiasis y la pancreatitis ha resuelto, se realiza colecistectomía con colangiografía transquirúrgica.

El uso de antibióticos está indicado en pacientes con pancreatitis severa cuando se desarrolla sepsis y/o falla orgánica múltiple, infección extrapancreática o posterior a necrosectomía. ${ }^{17,18}$ Los antibióticos indicados son carbapenémicos, por ejemplo imipenem 1-4 g/ día en tres o cuatro dosis o quinolonas y metronidazol, debido a que tienen adecuada penetración. En caso de cultivos positivos para hongos se agrega fluconazol. El uso profiláctico no está indicado, ya que no disminuye la mortalidad ni la necesidad de tratamiento quirúrgico. ${ }^{19,20}$

Los procedimientos quirúrgicos en la pancreatitis aguda se reservan para resolver las complicaciones locales y evitar la recurrencia. El síndrome compartimental abdominal es una de las pocas indicaciones quirúrgicas en la fase temprana. La colecistectomía debe realizarse en pacientes con pancreatitis de etiología biliar. Su práctica se recomienda una vez que esté resuelta la pancreatitis aguda y que el procedimiento se efectúe dentro del mismo internamiento. ${ }^{16}$

\section{NECROSIS PANCREÁTICA}

La necrosis pancreática se presenta entre 10 y $20 \%$ de los pacientes con pancreatitis, tiene una 
mortalidad de 10 a 25\% y se agrega infección a la necrosis entre 40 y $70 \% .^{10,21}$ Inicia en las primeras 24-48 horas, la necrosis es secundaria a la activación de las enzimas pancreáticas dentro de los acinos pancreáticos, lo que provoca muerte celular y trombosis de la microvasculatura, lo anterior ocasiona la activación de forma local de citocinas y células proinflamatorias, dichas citocinas cruzan la circulación sistémica causando respuesta inflamatoria sistémica, insuficiencia respiratoria y falla orgánica múltiple en las primeras dos semanas de la enfermedad. Posterior a la segunda semana de evolución de la enfermedad se agrega infección, lo que conlleva una mayor morbilidad y mortalidad.

La necrosectomía es el procedimiento cuyo objetivo es remover el tejido necrótico. Dicho procedimiento debe postergarse lo más posible (3-4 semanas) para disminuir la morbilidad, mortalidad y ser lo más resolutivo. ${ }^{16}$ Los procedimientos deben ser lo menos invasivos, disminuir el traumatismo y a la vez ser lo más resolutivos posible, es decir retirar la mayor cantidad de tejido desvitalizado. Existen múltiples abordajes para realizar dicho procedimiento.

El abordaje percutáneo consiste en la colocación de un catéter guiado por tomografía al sitio de la necrosis. ${ }^{22}$ Posteriormente se hacen lavados con solución estéril permitiendo el drenaje del tejido necrótico. ${ }^{23}$ En un estudio (PANTER trial) se dividió la muestra en dos grupos, uno con abordaje abierto y el segundo con abordaje percutáneo, seguido en algunos casos de abordaje laparoscópico retroperitoneal (VARD), dicho estudio mostró menor presencia de falla orgánica, hernias, costos, días de estancia en terapia intensiva en el grupo de punción percutánea seguido de VARD (abordaje step up), además de este grupo se observó que un tercio de pacientes sólo requirió drenaje percutáneo. ${ }^{24,25}$

El abordaje endoscópico de la necrosis pancreática ha tomado un papel importante en los últimos años. El procedimiento consiste en identificar por ultrasonido endoscópico el sitio de necrosis; se coloca una guía, posteriormente se hacen dilataciones hasta un diámetro de $20 \mathrm{~mm}$, se drena o desbrida. ${ }^{26}$ Hasta en $95 \%$ se ha tenido éxito en un promedio de cuatro sesiones. Las complicaciones que surgen son neumoperitoneo $1 \%$, sangrado $12 \%$, perforación $6 \%$ y fístula $5 \%$.
Tiene una mortalidad de $6 \%$ dependiendo de la serie. Para llevar a cabo dicho procedimiento se requiere que la pared de la necrosis sea menor de $10 \mathrm{~mm}$ y que no haya estructuras entre la pared del estómago y de la necrosis. ${ }^{27,28}$

En las últimas dos décadas se ha introducido el abordaje de mínima invasión con resultados favorables, este tipo de abordaje tiene menor respuesta inflamatoria secundaria al procedimiento. El abordaje abdominal ha demostrado una baja mortalidad de $6 \%$ y conversión de $3 \%$. No se recomienda este abordaje en pacientes graves, con antecedentes quirúrgicos abdominales y con otras comorbilidades. El abordaje puede ser trasgástrico, a través del saco menor, infracólico, retrocólico y retrogástrico. La principal complicación que se ha presentado es la fístula pancreática. ${ }^{29}$ El abordaje VARD se realiza a través del retroperitoneal, lo que permite mantener la cavidad abdominal estéril, se efectúa posterior a punción percutánea en casos que no son resolutivos. El abordaje VARD se ha observado resolutivo en $30-83 \%$ de los casos en diversos estudios. Su mortalidad es de $4-33 \%$ y presenta complicaciones en $9-83 \%$, como sangrado, perforación de víscera hueca, fístula pancreática o entérica. ${ }^{30}$

El abordaje abierto ha demostrado ser una forma segura de necrosectomía, se ha detectado que la mortalidad para procedimientos de mínima invasión es de $11.8 \%$, mientras que para procedimientos abiertos es de $16.8 \%{ }^{31}$ Existen diversas técnicas de necrosectomía abierta: lavado continuo, empaquetamiento, reexploración programada y lavado cerrado. El manejo debe ser multidisciplinario e individualizado para cada paciente y el abordaje debe determinarse por el grado de infección, la respuesta inflamatoria del paciente y la extensión de la necrosis. ${ }^{32}$

\section{REFERENCIAS}

1. Bradley EL. Surgery of acute pancreatitis. The American Journal of Surgery. 2007; 194: s20-s23.

2. Tenner S, Baillie J, De Witt J, Swaroop VS. American College of Gastroenterology Guideline. Managment of acute pancreatitis. Am J Gastroenterol. 2013; 108: 1400-1415.

3. Ratia GT, Gutierrez CA, Granell VJ. Etiology of acute pancreatitis. Cent Eur J Med. 2014; 9: 530-542.

4. Sánchez-Lozada R, Camacho-Hernández MI, VegaChavaje RG, Garza-Flores JH, Campos-Castillo C, 
Gutiérrez-Vega R. Pancreatitis aguda: experiencia de cinco años en el Hospital General de México. Gac Med Mex. 2005; 141: 123-127.

5. UK Working Party on Acute Pancreatitis. UK guidelines for the management of acute pancreatitis. Gut. 2005; 54: 1-9.

6. Lankisch PG, Apte M, Banks PA. Acute pancreatitis. The Lancet. 2015; 386: 85-96.

7. Sarr MG, Banks PA, Bollen TL, Dervenis C, Gooszen $\mathrm{HG}$, Johnson CD, et al. The new revised classification of acute pancreatitis 2012. Surg Clin North Am. 2013; 93: 549-562.

8. Maheshwari R, Subramanian RM. Severe acute pancreatitis and necrotizing pancreatitis. Crit Care Clin. 2016; 32: 279-290.

9. Khaliq A, Dutta U, Kochhar R, Singh K. Management of acute pancreatitis: "PANCREAS" contains eight easy steps to remember the treatment OP. J Pancreas. 2010; 11: 492-493.

10. Haydock MD, Willms HR, Phillips A, Petrov MS. Fluid therapy in acute pancreatitis. Ann Surg. 2012; 00: 1-7.

11. Takeda K, Takada T, Kawarada Y, Hirata K, Mayumi T, Yoshida M, et al. JPN Guidelines for the management of acute pancreatitis: medical management of acute pancreatitis. J Hepatobiliary Pancreat Surg. 2006; 13: $42-47$

12. Schorn S, Ceyhan GO, Tieftrunk E, Friess H, Ekin DI. Pain management in acute pancreatitis. Pancreapedia. 2015; 15: 1-13.

13. Oláh A, Romics L Jr. Enteral nutrition in acute pancreatitis: a review of the current evidence. World J Gastroenterol. 2014; 20: 16123-1613.

14. Sathiaraj E, Murthy S, Mansard MJ, Rao GV, Mahukar S, Reddy DN. Clinical trial: oral feeding with a soft diet compared with clear liquid diet as initial meal in mild acute pancreatitis. Aliment Pharmacol Ther. 2008; 28: 777-781.

15. Alvarez-de Frutos V, Herranz-Antolín S, GarcíaMartínez MC. Nutrition in acute pancreatitis: a review. Cent Eur J Med. 2014; 9: 574-579.

16. Martin RF, Hein AR. Operative managment of acute pancreatitis. Surg Clin North Am. 2013; 93: 595-610.

17. Lim CL, Lee W, Liew YX, Tang SS, Chlebicki MP, Kwa AL. Role of antibiotic prophylaxis in necrotizing pancreatitis: a meta-analysis. J Gastrointest Surg. 2015; 19: 480-491.

18. Serrablo A, Tejedor L, Martínez J. Antibiotics in severe acute pancreatitis. Cent Eur J Med. 2014; 9: 565-573.

19. Howard TJ. The role of antimicrobial therapy in severe acute pancratitis. Surg Clin North Am. 2013; 93: 585-593.

20. Dellinger EP, Tellado JM, Soto NE, Ashley SW, Barie PS, Dugernier T, et al. Early antibiotic treatmente of severe acute necrotizing pancreatitis. Ann Surg. 2007; 245: 674-683.
21. Hany JC, Pappas TN. Necrotizing pancreatitis: diagnosis and managment. Surg Clin North Am. 2007; 87: 1431-1446.

22. Manoluk MD, VanSonneberg E. Interventional radiology for necrotiying pancreatitis. J Gastrointest Surg. 2011; 15: 1101-1103.

23. Sleeman D, Levi DM, Cheung MC, Rahnemai-Azar A, Parisek S, Casillas V, et al. Treatment of infected pancreatic necrosis. J Am Coll Surg. 2011; 212: 748-752.

24. Besselink MG, van Santvoort HC, Nieuwenhuijs VB, Boermeester MA, Bollen TL, Buskens E, et al. Minimally invasive "step-up approach" versus maximal necrosectomy in patients with acute necrotizing pancreatitis (PANTER trial). BMC Surgery. 2006; 6: 6.

25. Van Santvoort HC, Besselink MG, Bakker OJ, Sijbrand $\mathrm{HH}$, Boermeester MA, Dejong $\mathrm{CH}$, et al. A step-up approach or open necrosectomy for necrotizing pancreatitis. N Eng J Med. 2010; 363: 1491-1502.

26. Bahr MH, Davis BR, Vitale GC. Endoscopic managment of acute pancreatitis. Surg Clin North Am. 2013; 93: 563-584.

27. Fogel EL. Endoscopic pancreatic necrosectomy. J Gastrointest Surg. 2011; 15: 1098-1100.

28. Van Brunschot S, Fockens P, Bakker OJ, Besselink MG, Voermans RP, Poley JW, et al. Endoscopic transluminal necrosectomy in necrotising pancreatitis: a systematic review. Surg Endosc. 2014; 28: 1425-1438.

29. Mattruoka L, Parekh D. The minimally invasive approach to surgical managment of pancreatic diseases. Gastroenterol Clin North Am. 2012; 41: 77-101.

30. Van Brunschot S, Besselink MG, Bakker OJ, Boermeester MA. Video-assisted retroperitoneal debridment (VARD) of infectes necrotizing pancreatitis: an update. Curr Surg Rep. 2013; 1: 121-120.

31. Gou S, Xiong J, Wu H, Zhou F, Tao J, Liu T, et al. Five year cohort study of open pancreatic necrosectomy for necrotizing pancreatitis suggest it is safe and effective operation. J Gastrointest Surg. 2013; 17: 1634-1642.

32. Fernandez CC. Open pancreatic necrosectomy: indications in the minimally invasive era. J Gastrointest Surg. 2011; 15: 1089-1091.

Correspondencia:

Rodrigo Velázquez de la Vega

Villa Versalles Núm. 42,

Col. Paseos de las Palmas, 52787,

Mun. Huixquilucan, Estado de México.

Tel. 5529006609

E-mail: dr.rodrigovelazquez@gmail.com 